\title{
Effect of substrate nanopatterning on the growth and structure of pentacene films
}

\author{
May Ling Ng, ${ }^{1,2}$ A. B. Preobrajenski, ${ }^{2, *}$ A. A. Zakharov, ${ }^{2}$ A. S. Vinogradov, ${ }^{3}$ S. A. Krasnikov, ${ }^{4}$ \\ A. A. Cafolla, ${ }^{4}$ and N. Mårtensson ${ }^{1,2}$ \\ ${ }^{1}$ Department of Physics, Uppsala University, P.O. Box 530, 75121 Uppsala, Sweden \\ ${ }^{2}$ MAX-lab, Lund University, P.O. Box 118, 22100 Lund, Sweden \\ ${ }^{3}$ V. A. Fock Institute of Physics, St. Petersburg State University, 198504 St. Petersburg, Russia \\ ${ }^{4}$ School of Physical Sciences, Dublin City University, Glasnevin, Dublin 9, Ireland \\ (Received 20 November 2009; revised manuscript received 25 January 2010; published 25 March 2010)
}

\begin{abstract}
The effect of modulating the structure of thin pentacene $\left(\mathrm{C}_{22} \mathrm{H}_{14}\right)$ films by a nanopatterned inert substrate, known as hexagonal boron nitride nanomesh, is reported. Films of different thickness are grown and characterized by x-ray absorption, core-level photoemission, low-energy electron microscopy, microbeam low-energy electron diffraction, and scanning tunneling microscopy. Initially the pentacene molecules adsorb with the molecular plane lying flat on the substrate but they tend to flip up with increasing coverage, forming wellordered monolayer-thick islands of upright molecules with low nucleation density. The herringbone packing of the upright molecules is observed with scanning tunneling microscopy. The electronic structure of the adsorbed molecules is very similar to that of the gas-phase pentacene, implying weak interaction with the substrate and between the molecules. The periodic corrugation of the substrate surface causes the monolayer of upright pentacene molecules to form two different coincidence superstructures. The lattice parameters of the pentacene unit cell for each of these two substrate-induced domains are determined from the microdiffraction patterns. Both domains can occur in several equivalent configurations, thus resulting in a number of twins with a typical size of a few micrometers. The first monolayer grows in a layer-by-layer mode until it is completed while the second monolayer forms diffusion-limited fractal islands. Upon annealing, the pentacene films are thermally stable up to approximately $80{ }^{\circ} \mathrm{C}$ and thereafter the onset of desorption is observed.
\end{abstract}

DOI: 10.1103/PhysRevB.81.115449

PACS number(s): 81.07.Pr, 68.55.-a, 68.37.Nq, 68.37.Ef

\section{INTRODUCTION}

The structure of thin films of organic molecules is critically important for their performance as active layers in organic semiconductor devices. By exerting control over the molecular orientation and packing, it is possible to tune the electronic structure of these films in order to facilitate charge injection and maximize electron mobility. Pentacene molecules $\left(\mathrm{Pn}, \mathrm{C}_{22} \mathrm{H}_{14}\right)$ are among the most promising candidates for organic electronics because they crystallize in a well-ordered structure with exceptionally high charge-carrier mobility comparable to that of amorphous silicon. ${ }^{1,2}$ The main factors determining the molecular orientation and the long-range ordering in Pn films are the electronic structure and the symmetry of the substrate surface ${ }^{3}$ as well as the growth kinetics. ${ }^{4}$

In particular, the substrate surface plays a crucial role in dictating the orientation of the Pn molecules. In fact, the molecular orientation of thin pentacene films has been studied on a wide variety of surfaces, e.g., on $\mathrm{Ag},{ }^{4,5} \mathrm{Cu}^{6-8} \mathrm{C}_{60}$ film, ${ }^{9} \mathrm{Bi}(0001)$ film, ${ }^{10,11}$ highly oriented pyrolytic graphite (HOPG), ${ }^{12} \mathrm{Si}$ and $\mathrm{SiO}_{2},{ }^{13-15} \mathrm{Si}(111)-(\sqrt{3} \times \sqrt{3}) R 30^{\circ}-\mathrm{Ag}$ surface, ${ }^{16,17} \mathrm{Si}(111)-(4 \times 1)$-In surface, ${ }^{18}$ an organic selfassembled monolayer (ML) ${ }^{19}$ etc. From these studies, it is apparent that Pn may grow in two possible molecular orientations, i.e., with the molecules either lying or standing on the substrate. In the case of a considerable chemical bonding with the substrate the molecules are typically lying flat while on more inert surfaces the weak van der Waals Pn-Pn interaction dominates, thus shaping the film into a bulklike herringbone structure formed by the standing molecules. How- ever, the exact morphology of the Pn films can vary significantly depending on the bonding to the surface. ${ }^{20}$

Another possible route toward reshaping the structure of the molecular film in a controllable way is to use a nanopatterned substrate with a periodic variation in the electrostatic potential along the surface. In particular, inert substrates with a strong periodic corrugation may be promising as templates for producing films with a desired motif because weakly bonded self-assembled molecular monolayers can be easily affected by lateral inhomogeneities. In addition, inert substrates are often nonmetallic or weakly metallic, thus they are better suited to serve as potential gate dielectrics.

An interesting substrate for studying the effect of nanopatterning on the structure of thin molecular films is a monolayer of hexagonal boron nitride (h-BN) periodically corrugated on $\mathrm{Rh}(111)$ or $\mathrm{Ru}(0001)$, often referred to as $\mathrm{h}-\mathrm{BN}$ nanomesh. According to previous studies, h-BN strongly chemisorbs on the lattice-mismatched $\mathrm{Rh}(111)$ and $\mathrm{Ru}(0001)$ surfaces forming a corrugated atomic layer with alternating bonding (pores) and nonbonding (wires) sites in a hexagonal pattern, with a lateral period of approximately $3.2 \mathrm{~nm} .{ }^{21-25}$ On $\mathrm{Rh}(111)$ the corrugation amplitude of the h-BN monolayer is calculated to be close to $0.55 \AA$ (Ref. 24) and varies for other lattice-mismatched transition-metal (TM) surfaces depending on their reactivity. ${ }^{26}$ The TM-supported h-BN monolayer in general represents a very inert substrate due to the rather low density of adsorption-induced h-BN gap states. ${ }^{27,28}$ Therefore, the interaction between the deposited molecules and $\mathrm{h}-\mathrm{BN} / \mathrm{Rh}$ is expected to be weak, thus increasing the probability for the corrugation to influence the structure of molecular films.

In this work we study the in situ growth and morphology 
of Pn films on $\mathrm{h}-\mathrm{BN} / \mathrm{Rh}(111)$ with angle-dependent nearedge $\mathrm{x}$-ray absorption fine-structure (NEXAFS) spectroscopy, core-level photoelectron spectroscopy (PES), lowenergy electron microscopy (LEEM), microbeam low-energy electron diffraction ( $\mu$-LEED), and scanning tunneling microscopy (STM). The main question to address here is whether the Pn films can adjust their morphology to match the periodic corrugation of the substrate. We demonstrate that such an adjustment does occur. In addition, we describe the symmetry and lattice parameters of the films, follow the variations in the growth mode as a function of Pn coverage, and discuss the thermal stability of the grown films. For comparison, we also study the growth of Pn on the bare $\mathrm{Rh}(111)$ surface.

\section{EXPERIMENTAL DETAILS}

The characterization of the Pn films grown on the h-BN nanomesh was performed in three stages, namely, by corehole spectroscopies, LEEM/ $\mu$-LEED, and STM. All samples were prepared in situ in the respective experimental chambers. A Rh(111) single crystal was used as the substrate for the growth of h-BN followed by Pn deposition. The procedures of cleaning the Rh crystal and the deposition of h-BN on $\mathrm{Rh}$ are described elsewhere. ${ }^{25}$ The quality of the h-BN overlayer formed on Rh was examined by both LEED and PES. Subsequently, pentacene (Sigma-Aldrich) was thermally evaporated from a Knudsen cell on the h-BN/Rh surface at room temperature (RT). The same Pn evaporation procedure was repeated on the clean $\mathrm{Rh}(111)$ surface for comparison. To calibrate the deposition rate of the Pn source, a reference sample with a monolayer of graphite (graphene) grown by chemical vapor deposition on $\mathrm{Rh}(111)$, as described in Ref. 29, was used. The intensity of the C $1 s$ PE signal from the Pn molecules in the initial stage of deposition can be easily transformed into the number of $\mathrm{C}$ atoms by comparing with the $\mathrm{C} 1 s$ intensity from the graphene sample for which the $\mathrm{C}$ atom density is well known. $1 \mathrm{ML}$ of lying or standing Pn molecules is defined throughout this paper following the work by Chen et al. ${ }^{12}$ on the Pn growth on HOPG, as illustrated schematically in Fig. 1.

The samples were characterized by angle-dependent $\mathrm{C} 1 \mathrm{~s}$ NEXAFS and C $1 s$ PES at beamline D1011, MAX-lab, Lund University. The photon energy resolution for the $\mathrm{C} K$ edge was $75 \mathrm{meV}$ while the kinetic-energy resolution for the $\mathrm{C} 1 \mathrm{~s}$ PES spectra was $125 \mathrm{meV}$. X-ray absorption measurements were performed in the partial electron yield mode with the retardation voltage $U$ of $-100 \mathrm{~V}$ in order to improve the signal-to-background ratio. The NEXAFS spectra were normalized to the spectrum from the pristine h-BN/Rh substrate taken in the same energy range. The $\mathrm{C} 1 s \mathrm{PE}$ spectra were analyzed by peak fitting with the program FITXPS. ${ }^{30}$

The growth of pentacene from sub-ML to over $1 \mathrm{ML}$ on $\mathrm{h}-\mathrm{BN} / \mathrm{Rh}(111)$ and clean $\mathrm{Rh}(111)$ was also examined with LEEM and $\mu$-LEED at beamline I311, MAX-lab, Lund University. The nucleation density and the Pn growth mode were determined from the LEEM data while the $\mu$-LEED patterns were used to reveal the symmetry and rotational alignment of the films on the substrate on the submicrometers scale. Typi-

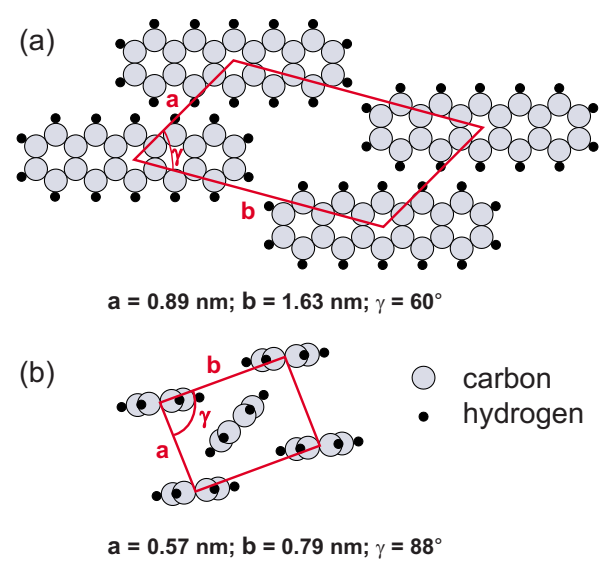

FIG. 1. (Color online) Schematics of the packing for (a) lying down and (b) upright pentacene molecules on HOPG (Ref. 12) used in the present work to define $1 \mathrm{ML}$ thickness of $\mathrm{Pn}$ on h-BN nanomesh.

cally, sample areas of $400 \mathrm{~nm}$ were probed in the $\mu$-LEED mode in order to prevent overlapping patterns from different domains. Prolonged electron bombardment caused structural damages to the Pn adlayer, especially in the $\mu$-LEED mode. To avoid this, the probed area was changed at the slightest sign of film degradation.

The STM experiments were performed at room temperature, using a commercial instrument (Omicron Nanotechnology $\mathrm{GmbH}$ ), in an ultrahigh-vacuum system consisting of an analysis chamber and a preparation chamber. An electrochemically etched polycrystalline tungsten tip was used to record STM images in constant current mode. The voltage $V$ corresponds to the sample bias with respect to the tip. No drift corrections have been applied to any of the STM images presented in this paper. All STM pictures were processed using the WSXM software. ${ }^{31}$

\section{RESULTS AND DISCUSSION}

Angle-dependent NEXAFS is a powerful tool commonly used in determining molecular orientation at surfaces. The $\mathrm{C} K$-edge NEXAFS spectra in Fig. 2 comprise three main regions, representing electron transitions from $\mathrm{C} 1 s$ to the lowest unoccupied molecular orbital (LUMO), LUMO+1 (both with $\pi$ symmetry), and to the continuum $\sigma$ states. ${ }^{19}$ The $\pi^{*}$ resonance manifold results from transitions from the six inequivalent $\mathrm{C} 1 s$ core levels of the Pn molecule to the LUMO and LUMO +1 , respectively. ${ }^{32}$

Figure 2(a) clearly shows that at the very beginning of the Pn deposition (in this case, at the coverage corresponding to $0.07 \mathrm{ML}$ of flat-lying Pn), there are only weak $\pi^{*}$ resonances observed at normal incidence while at grazing angles these signals become significantly stronger. This implies that most $\mathrm{Pn}$ molecules are lying flat on the $\mathrm{h}-\mathrm{BN}$ nanomesh at this low coverage. As more Pn is deposited onto the substrate, the situation gradually reverses; only a very weak $\pi^{*}$ resonance intensity is observed at grazing incidence with a stronger signal observed at normal incidence, as shown in Fig. 2(b) for a Pn coverage corresponding to $0.35 \mathrm{ML}$ of upright mol- 


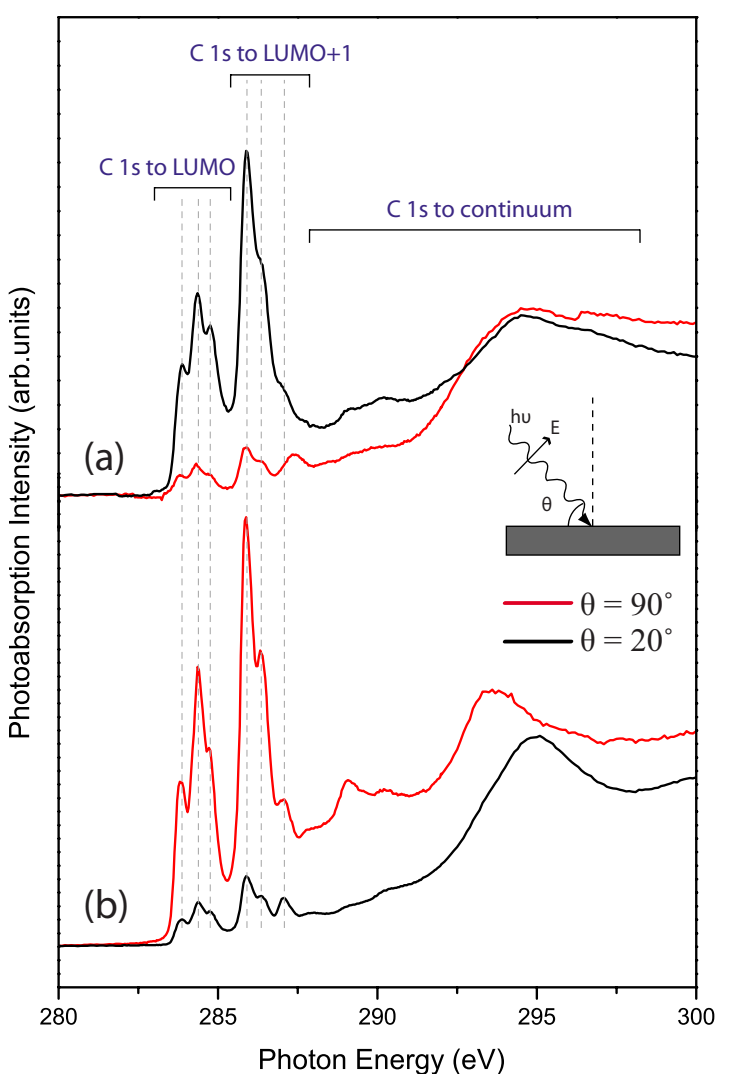

FIG. 2. (Color online) Angle dependent C $1 s$ NEXAFS spectra, where $\theta$ is the angle between the photon light polarization vector and the substrate surface normal. The black and red curves are for $\theta$ equal to $20^{\circ}$ (grazing incident) and $90^{\circ}$ (normal incident) relative to the substrate, respectively. (a) $0.07 \mathrm{ML}$ of flat lying and (b) 0.35 ML of upright Pn on h-BN/Rh.

ecules. From these NEXAFS results, it is obvious that the Pn molecules tilt upward with increasing Pn coverage. The onset of tilting takes place at around $0.2-0.3 \mathrm{ML}$ of flat-lying Pn (not shown). It is plausible to associate the delay in the onset of tilting with the lack of adjacent molecules to form the seeds of this upright phase in the beginning of the growth process. These findings are in accord with the computational study of submonolayer growth of Pn by Choudhary et al. $;^{20}$ once a critical nucleus of Pn is formed under favorable conditions, the molecules tend to flip up from flat lying to upright orientation at the onset of aggregation resulting in the formation of a crystalline phase.

It should be noted that the intensity of the $\pi^{*}$ resonances does not drop fast enough with the incident angle going off normal to account for a perfectly vertical orientation of the molecules in the film and a rather strong signal is visible even at $20^{\circ}$ [Fig. 2(b), black curve). This may be a result of the Pn molecules not standing strictly upright but instead inclined at a small angle to the surface normal, i.e., closely imitating bulk Pn, where the unit cell angles $\alpha$ and $\beta$ are $101.9^{\circ}$ and $112.6^{\circ}$, respectively. ${ }^{33}$ An experimental determination of these angles by NEXAFS is not possible in our case because several Pn domains contribute to the total signal, as will be shown below.

The overall shape of the C $1 s$ NEXAFS is similar to that recorded from the $\mathrm{Pn}$ molecules in the gas phase, ${ }^{32}$ indicating

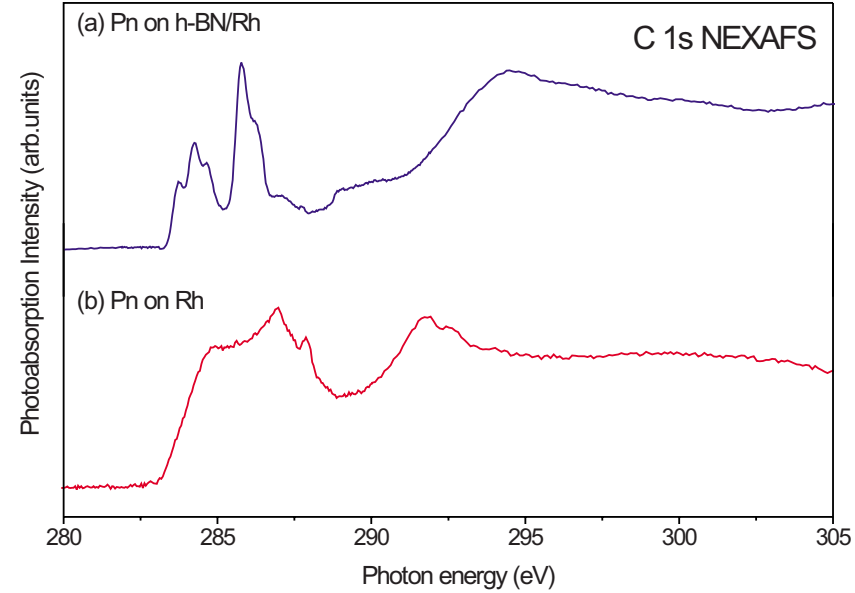

FIG. 3. (Color online) C $K$-edge NEXAFS of sub-ML of Pn (a) on h-BN/Rh and (b) on Rh with photons incident on the sample surface at an angle of $50^{\circ}$ relative to the substrate.

that there is no evident chemical interaction between Pn and the h-BN layer. However, the $\pi^{*}$ resonances are broader and less distinctive in the case of the flat-lying molecules [Fig. 2(a)] than for the upright orientation (b), which in fact can be interpreted as a manifestation of some (very weak) chemical $\pi-\pi$ bonding as long as the molecular plane is oriented parallel to the h-BN surface. This interaction cannot be compared with the chemisorption of Pn molecules on strongly reactive substrates, which may cause structural distortions of the molecule and thus inhibit the crystal growth. ${ }^{34}$ The case of strong chemisorption is demonstrated in Fig. 3, comparing the C $1 s$ NEXAFS spectra from lying down Pn molecules on $\mathrm{Rh}(111)$ with (a) and without (b) the h-BN interlayer. Evidently, for Pn on $\mathrm{Rh}$ there is a severe smearing of the $\pi^{*}$ resonances as a result of orbital mixing between the C $2 p(\pi)$ states of $\mathrm{Pn}$ and the $\mathrm{Rh} 4 d$ states, i.e., clear evidence for chemisorption.

Figure 4 shows the $\mathrm{C} 1 s$ PE spectra where $\mathrm{C} 1-\mathrm{C} 6$ represent the six inequivalent $\mathrm{C}$ atoms in the $\mathrm{Pn}$ molecule. The sequence of the components in descending binding energy (BE) is the following: $\mathrm{C} 2$ and $\mathrm{C} 4-\mathrm{C} 5$ and $\mathrm{C} 6-\mathrm{C} 1$ and $\mathrm{C} 3$. The signals $\mathrm{C} 2+\mathrm{C} 4, \mathrm{C} 5+\mathrm{C} 6$, and $\mathrm{C} 3+\mathrm{C} 1$ are each fitted with a single component due to the very small differences in BEs within each pair. This fit of the C $1 s$ PE spectra is in fair agreement with the theoretical calculations for gas-phase $\mathrm{Pn}^{32}$ and previous experimental studies of thin Pn films. ${ }^{35}$

Since there is no significant chemical interaction between the h-BN substrate and the Pn molecules, the thicknessrelated variation in the $\mathrm{C} 1 s$ PES can be attributed entirely to the gradual rearrangement of the molecules from the parallel to upright orientation. At low coverage [Fig. 4(a)] the molecules are mostly lying flat and do not form bonds with either the h-BN substrate or neighboring molecules. Therefore, the intensity of each components in the $\mathrm{C} 1 \mathrm{~s}$ spectrum is determined simply by the number of equivalent $\mathrm{C}$ atoms in the molecule. Indeed, this is the case for the spectrum in Fig. 4(a), where the intensities of components $\mathrm{C} 2+\mathrm{C} 4, \mathrm{C} 5+\mathrm{C} 6$, and $\mathrm{C} 3+\mathrm{C} 1$ are in the ratio $4: 4: 3$, thus providing additional evidence that the molecules are lying flat at very low coverage. Note also that a weak component due to some (small) 


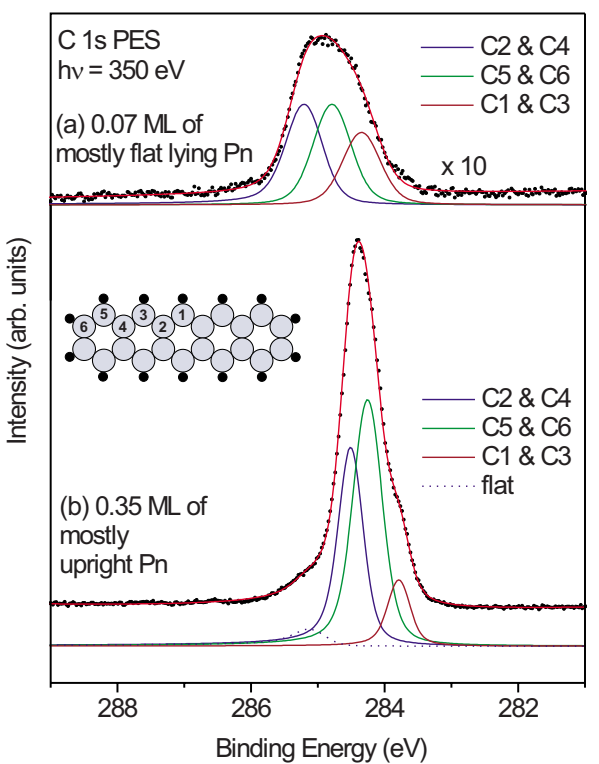

FIG. 4. (Color online) C $1 s$ PES of (a) 0.07 ML of flat lying and (b) $0.35 \mathrm{ML}$ of upright Pn molecules on h-BN/Rh(111). The photon excitation energy is $350 \mathrm{eV}$. Inset: schematic drawing of the Pn molecule with the nonequivalent $\mathrm{C}$ atoms numbered from 1 to 6 .

amount of the upright phase may be additionally fitted into the spectrum at the BE of $\sim 283.8 \mathrm{eV}$ [omitted in Fig. 4(a)].

As the molecules start to rearrange through interactions with the adjacent molecules, the component $\mathrm{C} 5+\mathrm{C} 6$ dominates the C $1 s$ PES spectrum, as can be seen in Fig. 4(b). Naturally, for standing Pn molecules, C5 and C6 are the most exposed $\mathrm{C}$ atoms while the others $(\mathrm{C} 1-\mathrm{C} 4)$ are more concealed between the neighboring molecules, with a corresponding attenuation of their photoelectron contribution. The weakest is the signal $\mathrm{C} 1+\mathrm{C} 3$ since the corresponding $\mathrm{C}$ atoms are the least exposed in the case of upright orientation. These data support the earlier conclusion that the molecules tend to flip up with increasing coverage. Since the upright monolayer is not yet complete, a component labeled "flat" was included in the fit to account for the residual isolated Pn molecules lying flat on the $\mathrm{h}-\mathrm{BN}$ nanomesh. It is also evident from Fig. 4 that the entire $\mathrm{C} 1 s$ PE spectrum is shifted by approximately $0.5 \mathrm{eV}$ to lower BEs with increasing Pn coverage. This can be attributed to a change in the work function induced by the molecular film (the BEs are measured relative to the Fermi level).

LEEM studies were performed in order to investigate the growth mode and the crystalline order of the Pn films. The images in Figs. 5(a) and 5(b) show a typical 1 ML thick island composed of upright Pn molecules grown on h-BN/ $\mathrm{Rh}(111)$ at $\mathrm{RT}$. The reversed contrast observed for two close electron energies provides clear evidence that the island is not amorphous but crystalline. Only the upright phase can be crystalline in this system, as can be judged from the analysis of the $\mu$-LEED patterns and as discussed below. The nucleation density is very low resulting in a typical grain size of several tens of micrometers at RT. This illustrates that the molecular mobility is high on the h-BN nanomesh, despite the periodic corrugation. In this sense the growth behavior of Pn on the h-BN substrate is similar to that observed on an-
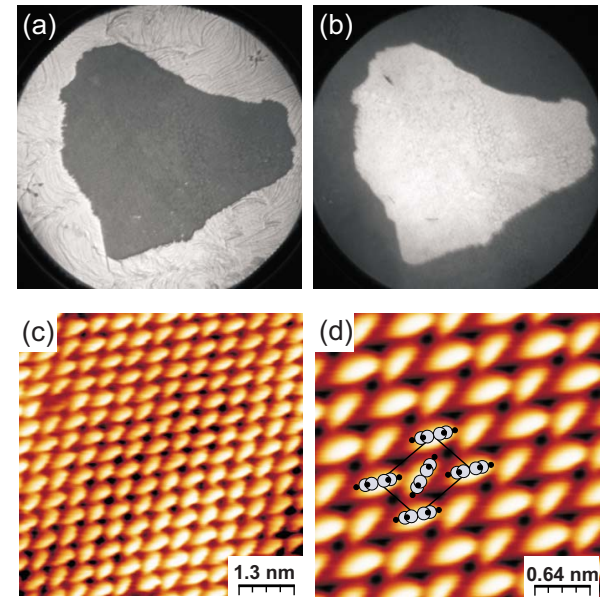

FIG. 5. (Color online) [(a) and (b)] LEEM images of a typical 1-ML-thick island of upright Pn grown on the h-BN/Rh(111) substrate. Field of view is $20 \mu \mathrm{m}$; the beam energy is (a) $3.4 \mathrm{eV}$ and (b) $5.7 \mathrm{eV}$. (c) STM image from a similar Pn island demonstrating an upright orientation and a herringbone packing $\left(V_{S}=-1.25 \mathrm{~V}, I\right.$ $=0.1 \mathrm{nA}$ ). (d) Similar to (c) but averaged over the unit cell; a single Pn unit cell is highlighted.

other inert substrate, $\mathrm{Bi}(0001)$, as shown by Sadowski et al. ${ }^{10,11}$

The herringbone packing of the upright Pn monolayer is evident in the STM studies and is illustrated in Figs. 5(c) and 5(d). Pn molecules appear as bright elongated protrusions on these images. Two translationally inequivalent molecules are located at the $(0,0)$ and $(1 / 2,1 / 2)$ points of the surface unit cell, with an angle $\Theta$ between their molecular planes. It should be noted that scanning over the film of upright pentacene molecules is difficult due to the very pliable nature of the film, which can be easily affected by the tip. This may result in distortion of the unit cells in the STM images, including distortions in $\Theta$, the herringbone angle. An additional complication arises due to the coexistence of different domains with similar unit-cell parameters, as shown below. Therefore, it is more reliable to determine the unit-cell parameters by diffraction techniques. Furthermore, the effect of the substrate nanopatterning on the unit-cell parameters can best be determined by diffraction.

In fact, the impact of the nanomesh corrugation on the final structure of the Pn monolayer turns out to be very strong, as can be seen in the $\mu$-LEED patterns. Figure 6 displays the $\mu$-LEED patterns recorded with the same electron energy $(10 \mathrm{eV})$ from a $400 \mathrm{~nm}$ region on the pristine h-BN/Rh substrate and on a 1-ML-thick Pn island. As the electron beam is moved along the island, several different patterns are observed representing different domain structures. These patterns can be separated in two groups with principally different (nonequivalent) arrangement of the spots while within each group the patterns are caused by rotational and (in one case) reflection twins. The two essentially different structures are denoted " $\mathrm{A}$ " and "B" and their diffraction patterns are shown in Fig. 6 along with schematic of the corresponding reciprocal and real-space lattices. The diffraction patterns from the Pn domains reproduce the hexagonal reconstruction of the corrugated h-BN layer under- 

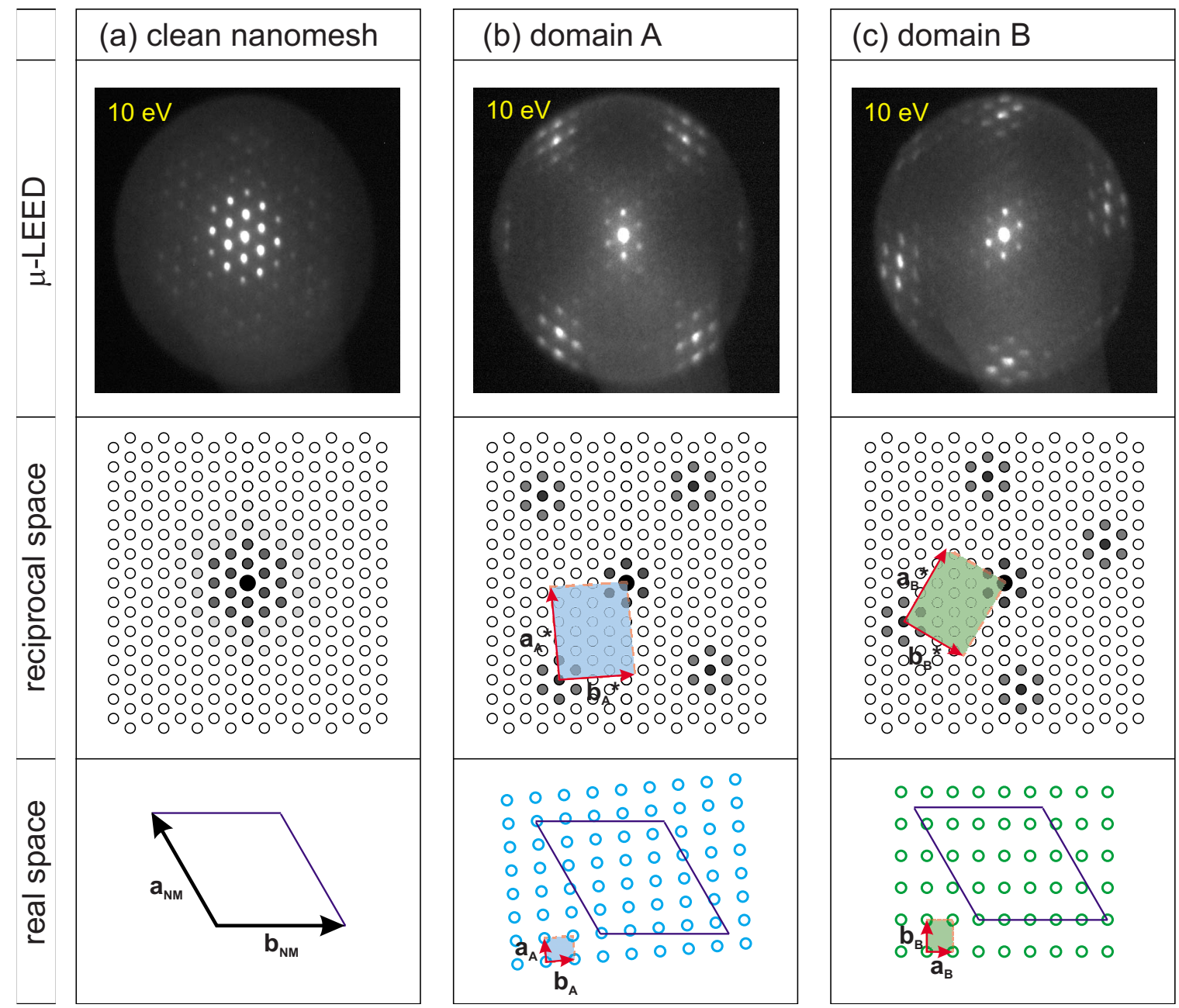

FIG. 6. (Color online) $\mu$-LEED patterns ( $V=10 \mathrm{eV}$, illumination area on sample is $400 \mathrm{~nm}$ ) and the corresponding schematic drawings of the reciprocal and real-space lattices for (a) pristine h-BN nanomesh on $\mathrm{Rh}(111)$, (b) domain A, and (c) domain B of 1-ML-thick Pn islands on h-BN/Rh. The rhombus highlights the unit cell of the underlying nanomesh, the Pn unit cells are shadowed in both reciprocal and real space. The real-space drawing illustrates the mutual relationship between the unit-cell parameters of the Pn film and the nanomesh substrate (two different coincidence lattices).

neath the film, resulting in coincidence patterns. The overlayer is forced by the substrate to match its structure to the hexagonal nanomesh lattice. This coincidence results in highlighting those nanomesh diffraction spots that correspond to the matched situations. The fact that there are two possible domain types should be attributed to a small difference in their formation energies. From the diffraction pattern analysis, the unit-cell parameters of these domains are determined in terms of those of the nanomesh unit cell. Based on the known parameters of the latter $\left[a_{\mathrm{NM}}=b_{\mathrm{NM}}=3.2 \mathrm{~nm}\right.$ and $\gamma=60^{\circ}$ (Ref. 22)], the geometries of the unit cells can be determined for both domain types, as shown in Fig. 6 and summarized in Table I. Due to the formation of coincidence lattices, the precision in estimating the lengths $a_{\mathrm{A}}\left(a_{\mathrm{B}}\right)$ and $b_{\mathrm{A}}\left(b_{\mathrm{B}}\right)$ is limited only by the precision of the corresponding parameter of the nanomesh while the angles are determined exactly. The lateral size of domains varies on the scale of several micrometers, as can be judged by moving the illumination aperture along the Pn islands.

In the case of domain B the two-dimensional (2D) unit cell is rectangular, resulting in three twin domains rotated by $\pm 120^{\circ}$ about the $c$ axis, which were observed experimentally. The surface unit cell for domain $\mathrm{A}$ is oblique, which implies that in addition to the three rotational twins it has to have another three twins for the changed direction of the $c$ axis (reflection twins). We cannot be sure we observed all of them, due to the limited number of domains sampled, but we have diffraction evidence for at least three twins for domain A.

From a comparison with the morphology of upright Pn monolayers on other substrates (see Table I) it is evident that the crystalline structure of these films is very pliable and easy to modify. Indeed, despite the fact that the herringbone motif of the bulklike Pn crystal is repeated in all such systems, the exact parameters of the unit cell can differ considerably. The same must be true for the herringbone angle, $\Theta$. Therefore, it seems plausible that the nanometer-scale periodic modulations of the substrate electrostatic potential can reshape the film structure and bring it into coincidence with the h-BN nanomesh.

The thermal stability of the 1 ML upright Pn films on h-BN/Rh was studied with LEEM. Figure 7 shows a continu- 
TABLE I. The lattice parameters of the Pn unit cell on different substrate surfaces.

\begin{tabular}{|c|c|c|c|c|}
\hline & \multirow[b]{2}{*}{ Type of Pn } & \multicolumn{3}{|c|}{ Lattice parameters } \\
\hline & & $\begin{array}{c}a \\
(\AA)\end{array}$ & $\begin{array}{c}b \\
(\AA)\end{array}$ & $\begin{array}{c}\gamma \\
(\mathrm{deg})\end{array}$ \\
\hline \multirow[t]{2}{*}{$\mathrm{Pn} / \mathrm{h}-\mathrm{BN} / \mathrm{Rh}$} & Domain A & 5.8 & 7.1 & 88.5 \\
\hline & Domain B & 6.4 & 7.9 & 90.0 \\
\hline \multirow[t]{2}{*}{ Pn/H-Si (Ref. 36) } & Type I & 6.02 & 7.62 & 90.0 \\
\hline & Type II & 5.98 & 7.56 & 90.3 \\
\hline \multirow[t]{2}{*}{ Pn/HOPG (Ref. 12) } & Lying down & 8.9 & 16.3 & 60 \\
\hline & Standing up & 5.7 & 7.9 & 88 \\
\hline Pn/Bi/Si (Ref. 11) & Standing up & 6.1 & 7.7 & 86 \\
\hline Bulk Pn (Ref. 33) & Crystal & 6.14 & 7.93 & 85.8 \\
\hline
\end{tabular}

ous Pn island on h-BN/Rh at RT (a) and the same island after annealing to $100{ }^{\circ} \mathrm{C}$ (b). Evidently, the Pn begins to desorb from the h-BN surface causing dark gray patches (bare $\mathrm{h}-\mathrm{BN} / \mathrm{Rh}$ ) on the previously light gray area (Pn island). The onset of the abrupt desorption is observed at approximately $80{ }^{\circ} \mathrm{C}$; this rather low desorption temperature is attributed to the weak substrate-adsorbate bonding. The temperatureinduced desorption from the islands is accompanied by a significant increase in the peak-to-background diffraction intensity ratio in the areas free from islands (compare insets in Fig. 7). This is a clear indication that originally there were flat-lying Pn molecules distributed randomly between the islands. These molecules desorb with increasing $T$ causing an improvement in the quality of diffraction pattern from the substrate.

As mentioned above, in the sub-ML regime Pn islands grow laterally in a layer-by-layer mode until the first ML is completed or almost completed. The RT nucleation density is greatly increased as the coverage exceeds $1 \mathrm{ML}$, resulting in Pn molecules forming fractal islands on top of the first Pn ML, as shown in Fig. 8(a). This growth mode resembles the regime of diffusion-limited aggregation (DLA), ${ }^{37}$ where the particles attach to the island as soon as they reach its border, without any rearrangement. The difference from the real DLA mode is that the nucleation density is high, resulting in
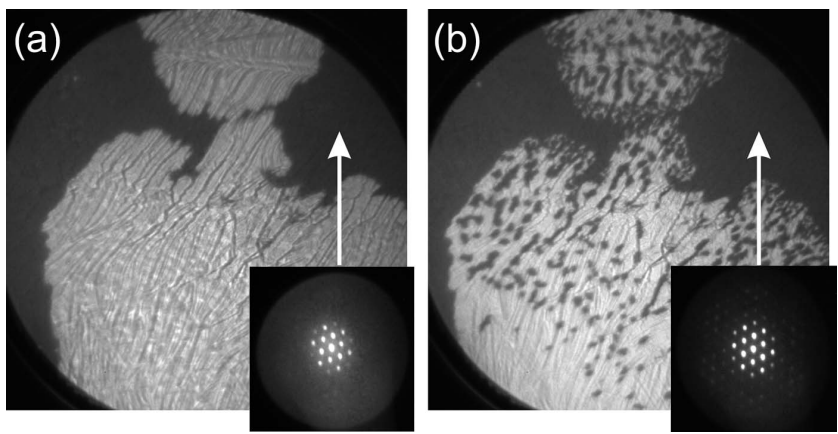

FIG. 7. Mirror mode LEEM of a sub-ML of Pn/h-BN/Rh at (a) room temperature and (b) $100{ }^{\circ} \mathrm{C}$. Field of view is $25 \mu \mathrm{m}$ for both images. The dark gray areas represent the uncovered h-BN/Rh while the light gray areas are regions covered with pentacene molecules. more compact islands than those observed in the typical DLA tree-shaped islands. The nucleation density can be considerably reduced by growing the second ML at elevated substrate temperatures resulting in increased molecular mobility, as shown in Fig. 8(b). In this case the island shapes are determined to a greater extent by diffusion. The reason for the different growth mode of the first and second monolayers lies in the considerably stronger electrostatic interaction of the Pn molecules with the already grown Pn film than with the bare h-BN monolayer. This interaction prevents molecules in the second Pn monolayer from finding the thermodynamically best position for attachment to the seed island by pinning it at the location where it first made contact with the island border. This shows again that the inert nature of the h-BN substrate is highly beneficial for the growth of well-ordered Pn films.

\section{CONCLUSIONS}

In the sub-ML to 1 ML regime, Pn molecules are weakly physisorbed in an upright orientation on a periodically corrugated h-BN monolayer grown on $\mathrm{Rh}(111)$. This first $\mathrm{Pn}$ monolayer grows in large, crystalline 2D islands in a layerby-layer mode with a herringbone arrangement of the molecules within unit cells. However, because individual molecules are lying flat on $\mathrm{h}-\mathrm{BN}$ and require the presence of neighboring Pn molecules to change their orientation, a criti-
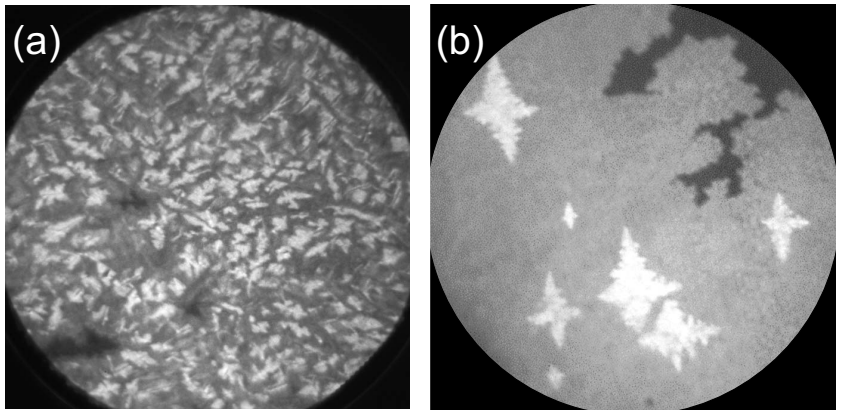

FIG. 8. LEEM images of the second Pn ML growing as fractal islands on top of the first ML (a) at RT and (b) at $80{ }^{\circ} \mathrm{C}$. For both images field of view is $20 \mu \mathrm{m}$ and $V=4.8 \mathrm{eV}$. 
cal coverage of $\mathrm{Pn}$ is required in order to initiate the formation of the upright layer. The nanopatterned h-BN/Rh(111) substrate strongly affects the parameters of the Pn unit cell resulting in the formation of two different coincidence lattices between the Pn film and the hexagonal h-BN/Rh surface. Both of these domains have twins rotated by $\pm 120^{\circ}$ while domain A can also have three reflection twins. Upon adsorption of the second monolayer, Pn molecules start to agglomerate into fractal islands as a result of the strong intermolecular interaction between the first and the second monolayers. This interaction causes significantly higher nucleation density and a growth mode resembling diffusionlimited aggregation. In the absence of the h-BN monolayer,
Pn molecules form strong bonds with the Rh(111) substrate. Despite the weak physisorption, a Pn monolayer grown on the h-BN nanomesh is thermally stable up to $80{ }^{\circ} \mathrm{C}$.

\section{ACKNOWLEDGMENTS}

We are grateful for the financial supports from the Swedish Research Council and the Russian Foundation for Fundamental Research (Grant No. 09-02-01278). A.A.C. and S.K. acknowledge support from Science Foundation Ireland through the Research Frontiers Programme (Grant No. 06/ RFP/PHY082). *alexeip@maxlab.lu.se

${ }^{1}$ D. Braga and G. Horowitz, Adv. Mater. (Weinheim, Ger.) 21, 1473 (2009), and references therein.

${ }^{2}$ C. D. Dimitrakopoulos and P. R. L. Malenfant, Adv. Mater. (Weinheim, Ger.) 14, 99 (2002), and references therein.

${ }^{3}$ G. E. Thayer, J. T. Sadowski, F. Meyer zu Heringdorf, T. Sakurai, and R. M. Tromp, Phys. Rev. Lett. 95, 256106 (2005).

${ }^{4}$ L. Casalis, M. F. Danisman, B. Nickel, G. Bracco, T. Toccoli, S. Iannotta, and G. Scoles, Phys. Rev. Lett. 90, 206101 (2003).

${ }^{5}$ Y. L. Wang, W. Ji, D. X. Shi, S. X. Du, C. Seidel, Y. G. Ma, H.-J. Gao, L. F. Chi, and H. Fuchs, Phys. Rev. B 69, 075408 (2004).

${ }^{6}$ S. Lukas, G. Witte, and Ch. Wöll, Phys. Rev. Lett. 88, 028301 (2001).

${ }^{7}$ O. McDonald, A. A. Cafolla, Z. Li, and G. Hughes, Surf. Sci. 600, 1909 (2006).

${ }^{8}$ E. Annese, C. E. Viol, B. Zhou, J. Fujii, I. Vobornik, C. Baldacchini, M. G. Betti, and G. Rossi, Surf. Sci. 601, 4242 (2007).

${ }^{9}$ A. Al-Mahboob, J. T. Sadowski, Y. Fujikawa, and T. Sakurai, Surf. Sci. 603, L53 (2009).

${ }^{10}$ J. T. Sadowski, T. Nagao, S. Yaginuma, Y. Fujikawa, A. AlMahboob, K. Nakajima, T. Sakurai, G. E. Thayer, and R. M. Tromp, Appl. Phys. Lett. 86, 073109 (2005).

${ }^{11}$ A. Al-Mahboob, J. T. Sadowski, T. Nishihara, Y. Fujikawa, Q. K. Xue, K. Nakajima, and T. Sakurai, Surf. Sci. 601, 1304 (2007).

${ }^{12}$ W. Chen, H. Huang, A. Thye, and S. Wee, Chem. Commun. (Cambridge) 2008, 4276.

${ }^{13}$ F.-J. Meyer zu Heringdorf, M. C. Reuter, and R. M. Tromp, Nature (London) 412, 517 (2001).

${ }^{14}$ G. Hughes, D. Carty, and A. A. Cafolla, Surf. Sci. 582, 90 (2005).

${ }^{15}$ R. Ruiz, B. Nickel, N. Koch, L. C. Feldman, R. F. Haglund, A. Kahn, and G. Scoles, Phys. Rev. B 67, 125406 (2003).

${ }^{16} \mathrm{Ph}$. Guaino, A. A. Cafolla, D. Carty, G. Sheerin, and G. Hughes, Surf. Sci. 540, 107 (2003).

${ }^{17}$ J. Teng, K. Wu, J. Guo, and E. Wang, Surf. Sci. 602, 3510 (2008).

${ }^{18}$ H. Zhang, Y. Li, B. Li, X. Fang, H. Ji, B. Wang, C. Zeng, and J. G. Hou, Surf. Sci. 603, L70 (2009).

${ }^{19}$ M. Chiodi, L. Gavioli, M. Beccari, V. Di Castro, A. Cossaro, L. Floreano, A. Morgante, A. Kanjilal, C. Mariani, and M. G. Betti, Phys. Rev. B 77, 115321 (2008).
${ }^{20}$ D. Choudhary, P. Clancy, R. Shetty, and F. Escobedo, Adv. Funct. Mater. 16, 1768 (2006).

${ }^{21}$ M. Corso, W. Auwärter, M. Muntwiler, A. Tamai, T. Greber, and J. Osterwalder, Science 303, 217 (2004).

${ }^{22}$ S. Berner, M. Corso, R. Widmer, O. Groening, R. Laskowski, P. Blaha, K. Schwarz, A. Goriachko, H. Over, S. Gsell, M. Schreck, H. Sachdev, T. Greber, and J. Osterwalder, Angew. Chem., Int. Ed. 46, 5115 (2007).

${ }^{23}$ A. Goriachko, Y. He, M. Knapp, and H. Over, Langmuir 23, 2928 (2007).

${ }^{24}$ R. Laskowski, P. Blaha, T. Gallauner, and K. Schwarz, Phys. Rev. Lett. 98, 106802 (2007).

${ }^{25}$ A. B. Preobrajenski, A. S. Vinogradov, M. L. Ng, E. Ćavar, R. Westerström, A. Mikkelsen, E. Lundgren, and N. Mårtensson, Phys. Rev. B 75, 245412 (2007).

${ }^{26}$ A. B. Preobrajenski, M. A. Nesterov, M. L. Ng, A. S. Vinogradov, and N. Mårtensson, Chem. Phys. Lett. 446, 119 (2007).

${ }^{27}$ A. B. Preobrajenski, S. A. Krasnikov, A. S. Vinogradov, M. L. Ng, T. Käämbre, A. A. Cafolla, and N. Mårtensson, Phys. Rev. B 77, 085421 (2008).

${ }^{28}$ R. Laskowski, P. Blaha, and K. Schwarz, Phys. Rev. B 78, 045409 (2008).

${ }^{29}$ A. B. Preobrajenski, M. L. Ng, A. S. Vinogradov, and N. Mårtensson, Phys. Rev. B 78, 073401 (2008).

${ }^{30}$ D. L. Adams, FITXPS (version 2.12), freely available from http:// www.sljus.lu.se/download.html

${ }^{31}$ I. Horcas, R. Fernández, J. M. Gómez-Rodríguez, J. Colchero, J. Gómez-Herrero, and A. M. Baro, Rev. Sci. Instrum. 78, 013705 (2007).

${ }^{32}$ M. Alagia, C. Baldacchini, M. G. Betti, F. Bussolotti, V. Carravetta, U. Ekström, C. Mariani, and S. Stranges, J. Chem. Phys. 122, 124305 (2005).

${ }^{33}$ R. B. Campbell, J. Monteath Robertson, and J. Trotter, Acta Crystallogr. 14, 705 (1961).

${ }^{34}$ R. J. Hamers, Nature (London) 412, 489 (2001).

${ }^{35}$ C. Baldacchini, F. Allegretti, R. Gunnella, and M. G. Betti, Surf. Sci. 601, 2603 (2007).

${ }^{36}$ S. Nishikata, G. Sazaki, J. T. Sadowski, A. Al-Mahboob, T. Nishihara, Y. Fujikawa, S. Suto, T. Sakurai, and K. Nakajima, Phys. Rev. B 76, 165424 (2007).

${ }^{37}$ T. A. Witten and L. M. Sander, Phys. Rev. Lett. 47, 1400 (1981). 